\title{
Usefulness of vessel plus surface classification system for the diagnosis of early gastric cancer after Helicobacter pylori eradication
}

\author{
Masaki Miyaokaa , Kenshi Yao a, Hiroshi Tanabec, Takao Kanemitsua , Kentaro Imamuraa, Yoichiro Onoa, \\ Kensei Ohtsua, Satoshi Ishikawaa, Toshiki Kojimaa , Rino Hasegawa a, Akikazu Hirano ${ }^{a}$, Go Ikezono , \\ Takashi Hisabe ${ }^{\mathrm{b}}$, Toshiharu Ueki ${ }^{\mathrm{b}}$, Atsuko Otac', Seiji Haraokac, Akinori Iwashitac
}

Fukuoka University Chikushi Hospital, Chikusino, Japan

\section{Abstract}

${ }^{a}$ Department of Endoscopy (Masaki Miyaoka, Kenshi Yao, Takao Kanemitsu, Kentaro Imamura, Yoichiro Ono, Kensei Ohtsu, Satoshi Ishikawa, Toshiki Kojima, Rino Hasegawa, Akikazu Hirano, Go Ikezono); ${ }^{\mathrm{b}}$ Department of Gastroenterology (Takashi Hisabe, Toshiyuki Ueki); 'Department of Pathology (Hiroshi Tanabe, Atsuko Ota, Seiji Haraoka, Akinori Iwashita), Fukuoka University Chikushi Hospital, Chikusino, Japan

Correspondence to: Kenshi Yao, MD, PhD, Department of Endoscopy, Fukuoka University Chikushi Hospital, 1-1-1 Zokumyoin, Chikushino City, Fukuoka 818-8502, Japan, e-mail: yao@fukuoka-u.ac.jp

Conflict of Interest: None

Received 26 September 2020; accepted 27 October 2020; published online 26 February 2021

DOI: https://doi.org/10.20524/aog.2021.0605

\section{Introduction}

In recent years, eradication of Helicobacter pylori (H. pylori) has been recommended for the primary prevention of gastric cancer [1]. Previous studies have shown that it is difficult to detect some cases of gastric cancer after $H$. pylori eradication using endoscopic diagnostic procedures including magnifying endoscopy with narrow-band imaging (ME-NBI), but most authors used surface structures alone as the main diagnostic marker [2,3].

We use the vessel plus surface (VS) classification system which employs both the microvascular pattern and microsurface pattern as markers for making a diagnosis of early gastric cancer by means of ME-NBI [4]. The VS classification system has been approved as a standard diagnostic system for 
magnifying endoscopy of gastric cancer based on domestic and international multi-society consensus [5]. The diagnostic criteria of gastric cancer for magnifying endoscopy according to the VS classification system are as follows: (1) presence of irregular microvascular pattern with a demarcation line; and/or (2) presence of irregular microsurface pattern with a demarcation line. However, no report has discussed the usefulness of magnifying endoscopy with both the microvascular and microsurface patterns as markers for diagnosing early gastric cancer after $H$. pylori eradication according to the VS classification system. Therefore, this study aimed to determine the usefulness of magnifying endoscopy based on the VS classification system for detecting gastric cancer after $H$. pylori eradication.

\section{Patients and methods}

\section{Patients}

The subjects were consecutive patients with early gastric cancer lesions treated with endoscopic submucosal dissection (ESD) in the Department of Endoscopy of Fukuoka University Chikushi Hospital between January 2016 and January 2017, who satisfied the following inclusion criteria, and did not meet the following exclusion criteria.

Inclusion criteria: (1) patients with early gastric cancer resected en bloc by ESD; (2) patients with a lesion diagnosed as early gastric cancer of differentiated type based on a histopathological examination of the resected specimen; (3) patients with a lesion for which endoscopic images at the maximum magnification were available from preoperative ME-NBI; and (4) patients with a lesion who are currently $H$. pylori-positive or in whom $H$. pylori was eradicated.

Exclusion criteria: (1) patients with a lesion diagnosed as gastric cancer of undifferentiated type or adenocarcinoma of fundic gland type based on a histopathological examination of the resected specimen; (2) patients with a lesion and insufficient histopathological examination of the resected specimen; and (3) patients with a lesion who were not previously infected with H. pylori or whose $H$. pylori infection status was unclear.

A patient was judged to be positive for $H$. pylori infection when at least 2 of the following 5 tests were positive: (1) serum immunoglobulin G antibody test; (2) ${ }^{13} \mathrm{C}$-urea breath test; (3) biopsy culture; (4) rapid urease test; and (5) histopathological finding. Successful H. pylori eradication was defined as the presence of a history of $H$. pylori eradication with a negative result of the ${ }^{13} \mathrm{C}$-urea breath test at the time of cancer diagnosis.

\section{Study procedures and endpoints}

All images of ME-NBI, obtained at the maximum magnification, were reviewed retrospectively by one endoscopist (M.M.), blinded to the final classification and diagnosis, to determine the presence or absence of the demarcation line, irregular microvascular pattern, and irregular microsurface pattern. The definition of the morphology for the demarcation line, irregular microvascular pattern, and irregular microsurface pattern in the previously published paper and guidelines were employed for this analysis [5-7]. Patients were divided into 2 groups: those with gastric cancer detected after $H$. pylori eradication (H. pylori-eradicated group) and those with $H$. pylori-positive gastric cancer (H. pylori-positive group). The positivity rates calculated for each of the aforementioned markers were compared between the 2 groups.

For endoscopy, an upper gastrointestinal tract magnifying endoscope (GIF-H260Z or GIF-H290Z, Olympus Co., Tokyo, Japan) with the endoscopy system Evis Lucera Elite (Olympus) was used. Structure enhancement was set at the B8 level during observation with ME-NBI [4]. Endoscopic observation was performed according to the procedure reported previously [8].

The primary endpoint was the difference in the positivity rate of the demarcation line, irregular microvascular pattern, or irregular microsurface pattern between the H. pylorieradicated group and the $H$. pylori-positive group. The secondary endpoints were as follows: (1) the difference in sensitivity between the irregular microvascular pattern and irregular microsurface pattern for detecting gastric cancer after $H$. pylori eradication; and (2) the difference in the frequencies of cancer containing a histological component of a very welldifferentiated adenocarcinoma.

\section{Statistical analysis}

Mann-Whitney $U$ test and Fisher's exact probability test were used to compare median values and frequencies, respectively, between the 2 groups. Bonferroni's multiple comparison test was used to perform comparisons among 3 groups (the numbers of the lesions in upper, middle and lower third parts in the stomach). SPSS, version 21J for Windows (SPSS, Chicago, IL, USA) was used to perform all statistical analyses. Differences were considered statistically significant at $\mathrm{P}<0.05$.

\section{Results}

\section{Patients}

One hundred forty lesions of 108 patients with early gastric cancer were treated with ESD in the Department of Endoscopy of our hospital between January 2016 and January 2017. Among these lesions, 85 were included in the analysis. These 85 lesions comprised 27 lesions in the $H$. pylori-eradicated group and 58 in the H. pylori-positive group (Fig. 1).

Clinical features of the lesions are shown in Table 1. There was no significant difference in sex, age, the lesion site, macroscopic type, or tumor size between the 2 groups. The median time (range) between eradication and ESD in the H. pylori-eradicated group was 37 (4-185) months. 
Positivity rates of the demarcation line, irregular microvascular pattern, and irregular microsurface pattern

A demarcation line was found in all lesions, producing a positivity rate of $100 \%$ in both groups (Table 2 ). The positivity rates of irregular microvascular pattern were also $100 \%$ (27/27) in the $H$. pylori-eradicated group and $100 \%(58 / 58)$ in the $H$. pylori-positive group (Table 2). However, the positivity rates of the irregular microsurface pattern were $78 \%(21 / 27)$ in the $H$. pylori-eradicated group and $98 \%(55 / 58)$ in the H. pylori-positive group. The positivity rate of the irregular microsurface pattern was statistically significantly lower in the $H$. pylori-eradicated group than in the $H$. pylori-positive group (Table 2).

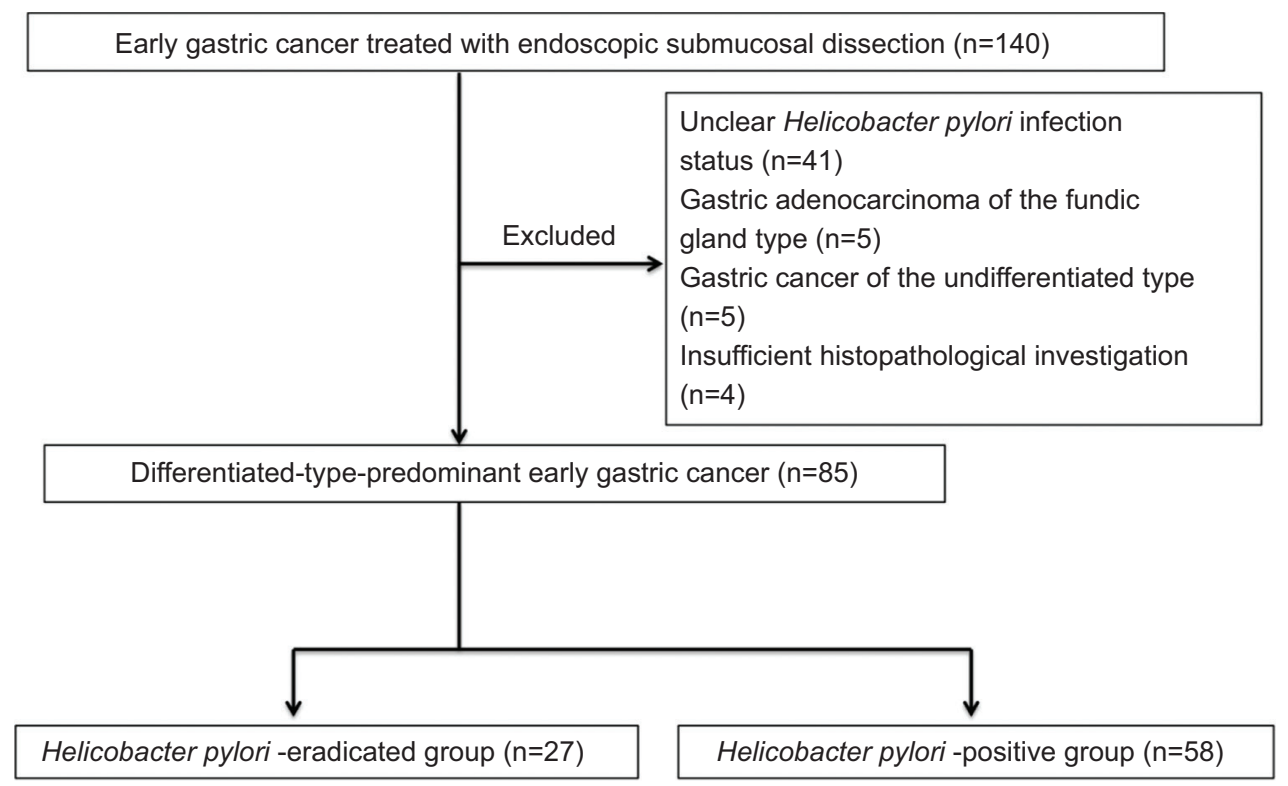

Figure 1 Flow diagram of patient enrolment

Table 1 Comparison of clinical characteristics of early gastric cancers between Helicobacter pylori (H. pylori)-eradicated and H. pylori-positive groups $(\mathrm{n}=85)$

\begin{tabular}{|c|c|c|c|c|}
\hline \multicolumn{2}{|l|}{ Characteristics } & \multirow{2}{*}{$\frac{\text { H. pylori-eradicated group } \mathrm{n}=27}{22(81 \%)}$} & \multirow{2}{*}{$\frac{\text { H. pylori-positive group } \mathrm{n}=58}{44(78 \%)}$} & \multirow{2}{*}{$\frac{\text { P-value }}{0.38^{1)}}$} \\
\hline Sex & Male & & & \\
\hline & Female & $5(19 \%)$ & $14(22 \%)$ & \\
\hline Median age, year ( & & $72(59-84)$ & $72(52-88)$ & $0.46^{2)}$ \\
\hline \multirow[t]{3}{*}{ Location } & Upper third & $4(15 \%)$ & $5(9 \%)$ & $0.56^{3)}$ \\
\hline & Middle third & $11(41 \%)$ & $21(36 \%)$ & \\
\hline & Lower third & $12(44 \%)$ & $32(55 \%)$ & \\
\hline \multirow[t]{2}{*}{ Macroscopic type } & Superficial elevated type & $2(7 \%)$ & $11(19 \%)$ & $0.14^{1)}$ \\
\hline & Superficial flat/depressed type & $25(93 \%)$ & $47(81 \%)$ & \\
\hline \multicolumn{2}{|c|}{ Median size, mm (range) } & $16(4-55)$ & $17(2-40)$ & $0.35^{2)}$ \\
\hline
\end{tabular}

1) Fisher's exact probability test; 2) Mann-Whitney U test; 3) Bonferroni's multiple comparison test

Table 2 Prevalence of demarcation line, irregular microvascular pattern, and irregular microsurface pattern $(\mathrm{n}=85)$

\begin{tabular}{lccc} 
& H. pylori-eradicated group $\mathrm{n}=27$ & H. pylori-positive group $\mathrm{n}=58$ & P-value \\
\hline Demarcation line & $27(100 \%)$ & $58(100 \%)$ & $>0.99$ \\
Irregular microvascular pattern & $27(100 \%)$ & $58(100 \%)$ & $>0.99$ \\
Irregular microsurface pattern & $21(78 \%)$ & $55(95 \%)$ & 0.03 \\
\hline
\end{tabular}

Fisher's exact probability test

H. pylori, Helicobacter pylori 


\section{Sensitivities of the irregular microvascular pattern and irregular microsurface pattern for diagnosing early gastric cancer after $\boldsymbol{H}$. pylori eradication}

The sensitivity of the irregular microvascular pattern as a marker was $100 \%$, whereas that of the irregular microsurface pattern as a marker was $78 \%$ (95\% confidence interval 62.3 93.1). The sensitivity of the irregular microsurface pattern was significantly lower than that of the irregular microvascular pattern $(\mathrm{P}=0.01)$.

\section{Frequency of cancer containing the histological component of a very well-differentiated adenocarcinoma}

A comparison of the 2 groups revealed that the frequencies of cancer containing the histological component of a very well-differentiated adenocarcinoma were $56 \%(15 / 27)$ in the H. pylori-eradicated group and $29 \%(17 / 58)$ in the $H$. pyloripositive group. Thus, gastric cancer containing the component of a very well-differentiated adenocarcinoma was statistically significantly higher in the $H$. pylori-eradicated group than in the H. pylori-positive group $(\mathrm{P}=0.02)$.

\section{Representative cases}

\section{Case 1: Gastric cancer detected after H. pylori eradication}

Identification of the irregular microvascular pattern alone was useful for making a correct diagnosis (Fig. 2). A flat,

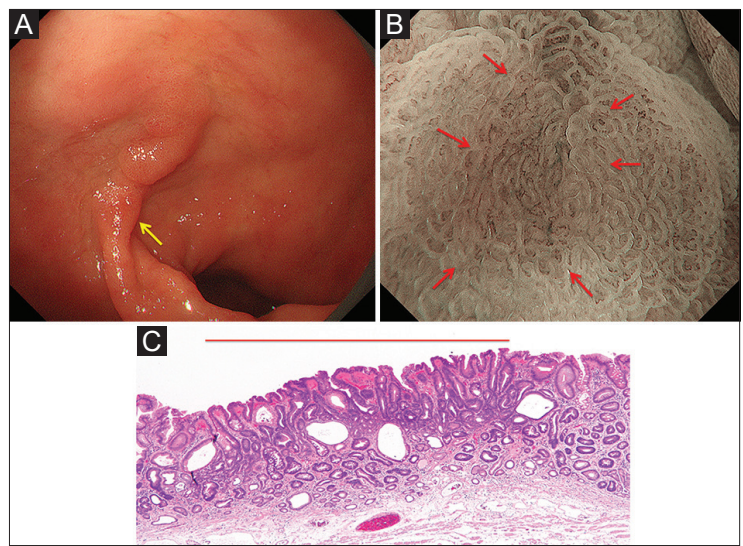

Figure 2 Case 1. Early gastric cancer detected after Helicobacter pylori eradication: A case of type IIa + IIb early gastric cancer in which the irregular microvascular pattern was useful for making a qualitative diagnosis. (A) Conventional endoscopic findings: There is a flat mucosal lesion measuring $15 \mathrm{~mm}$ in diameter in the anterior wall of the gastric antrum. The yellow arrow shows the area observed at a higher magnification. (B) Magnifying endoscopy with narrow-band imaging (ME-NBI) findings. According to the vessel plus surface classification system, the lesion has an irregular microvascular pattern and a regular microsurface pattern with a demarcation line. The red arrow shows the demarcation line. (C) Histopathological findings of the endoscopically resected specimen that correspond to the part observed by ME-NBI show a very well-differentiated adenocarcinoma. The red line shows the extent of the cancer (hematoxylin-eosin stain, $\times 40$ ) elevated-type lesion measuring about $15 \mathrm{~mm}$ in diameter, which had almost the same color tone as that of the surrounding area, was observed in the anterior wall of the gastric antrum under conventional white-light endoscopy. There was a slightly reddened flat part on the greater curvature side of the lesion (Fig. 2A). Using ME-NBI, the demarcation line was identified in terms of differences in microvascular architecture between the background noncancerous mucosa and cancerous mucosa. As for the microsurface structure inside the demarcation line, marginal crypt epithelia were curved with a uniform shape, symmetric distribution, and regular arrangement, leading to the identification of the regular microsurface pattern. However, as for the microvascular architecture, the microvessels showed various loop forms. No microvessels had identical forms, clearly showing morphological nonuniformity, and the arrangement was irregular, leading to the identification of the irregular microvascular pattern, typical of cancer (Fig. 2B). Fig. 2b shows the area observed by magnifying endoscopy. The resected specimen obtained from this area was histopathologically diagnosed as a very well-differentiated adenocarcinoma (Fig. 2C).

\section{Case 2: Gastric cancer detected after H. pylori eradication}

The irregular microvascular pattern and irregular microsurface pattern were found, and there were some areas
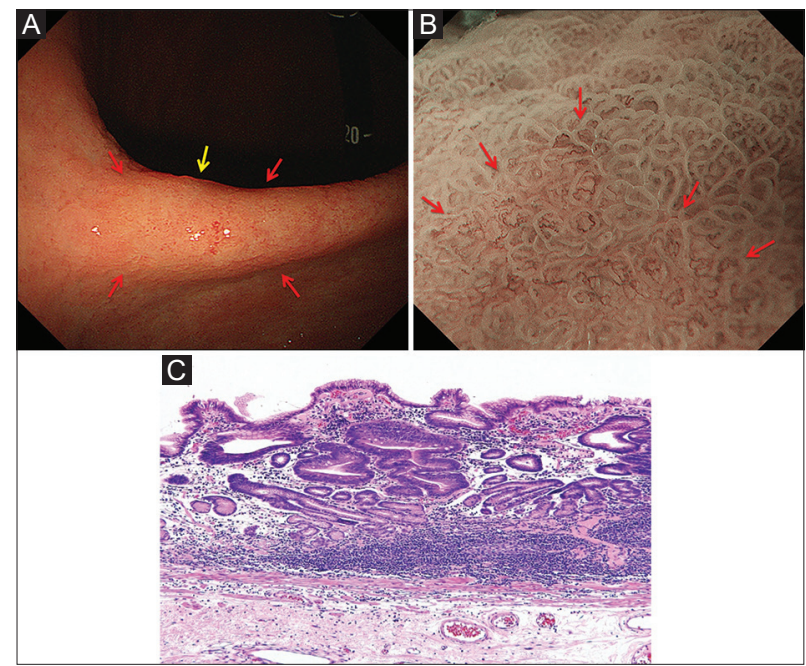

Figure 3 Case 2. Early gastric cancer detected after Helicobacter pylori eradication: A case of type 0 -IIb early gastric cancer in which the lesion measures $12 \mathrm{~mm}$ and has an irregular microvascular pattern and irregular microsurface pattern, and part of the lesion is covered by non-neoplastic epithelium. (A) Conventional white-light endoscopic findings. There is a flat mucosal lesion on the anterior wall side of the lesser curvature of the gastric angle. The lesion is a slightly discolored area. The yellow arrow shows the area observed at a higher magnification. (B) Magnifying endoscopy with narrow-band imaging findings. According to the vessel plus surface classification system, the irregular microvascular pattern and irregular microsurface pattern with a demarcation line are identified. The red arrow indicates the demarcation line. (C) Histopathological findings. The lesion consists of a well-differentiated adenocarcinoma with its superficial layer covered by non-neoplastic epithelium (hematoxylin-eosin stain, $\times 100$ ) 
where the tumor was covered by non-neoplastic epithelium (Fig. 3). A flat, slightly reddened lesion measuring $12 \mathrm{~mm}$ in diameter was found in the anterior wall of the lesser curvature of the gastric angle (Fig. 3A). A demarcation line was identified using ME-NBI in terms of differences in the microvascular architecture between the background noncancerous and cancerous mucosa. Regarding the microsurface structure within the demarcation line, marginal crypt epithelia were curved to oval, showing nonuniformity in shape. Their distribution was asymmetric, and their arrangement was irregular. Thus, the irregular microsurface pattern was identified. Concerning the microvascular architecture, the microvessels showed various loop forms, nonuniform shapes, irregular arrangement, and asymmetric distribution, leading to the identification of the irregular microvascular pattern (Fig. 3B). Histopathological examination of the resected specimen revealed that the lesion was a well-differentiated adenocarcinoma with the surface layer covered by nonneoplastic epithelium (Fig. 3C).

\section{Case 3: Gastric cancer detected after H. pylori eradication}

This patient had a typical irregular microvascular pattern and irregular microsurface pattern (Fig. 4). An irregularly shaped, depressed lesion measuring $7 \mathrm{~mm}$ in diameter, which had almost the same color tone as that of the surrounding area,

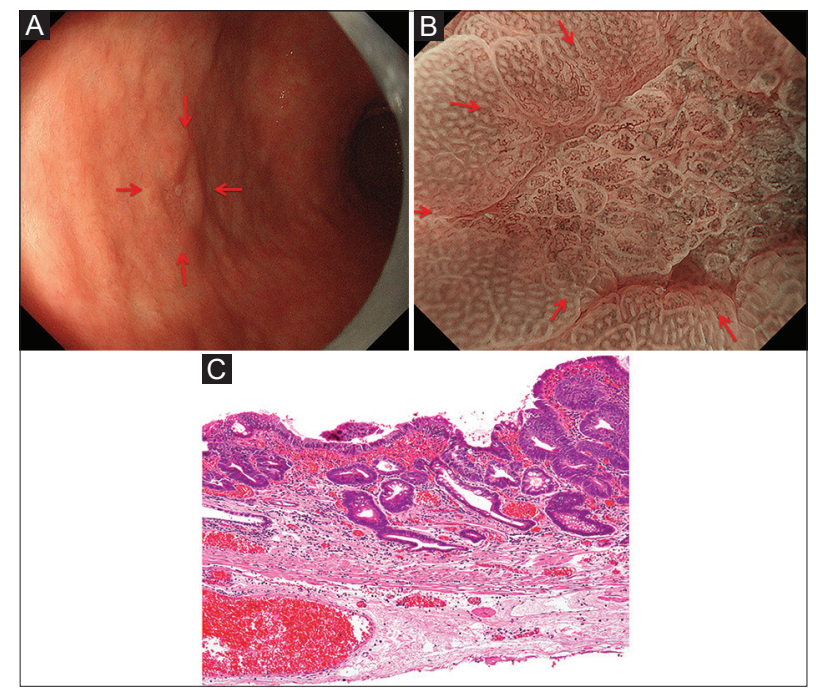

Figure 4 Case 3. Gastric cancer detected after Helicobacter pylori eradication: A case of type 0 -IIc early gastric cancer in which the lesion measures $7 \mathrm{~mm}$ and has a typical irregular microvascular pattern and irregular microsurface pattern. (A) Conventional white-light endoscopic findings. A slightly depressed lesion with an irregular margin (red arrow) is found in the anterior wall of the gastric antrum. (B) Magnifying endoscopy with narrow-band imaging findings. According to the vessel plus surface classification system, an irregular microvascular pattern and irregular microsurface pattern with a demarcation line are identified. The red arrow indicates the demarcation line. (C) Histopathological findings. The lesion consists of a well-differentiated adenocarcinoma (hematoxylin-eosin stain, $\times 100$ )

was observed in the anterior wall of the gastric antrum under conventional white-light endoscopy (Fig. 4A). A demarcation line was identified by ME-NBI in terms of differences between the microvascular architecture and microsurface structure. As for the microsurface structure inside the demarcation line, marginal crypt epithelia were curved to oval, showing nonuniform shapes, asymmetric distribution, and irregular arrangement, leading to the identification of the irregular microsurface pattern. Regarding the microvascular architecture, the microvessels were nonuniform in shape with irregular arrangement and asymmetric distribution, leading to the identification of the irregular microvascular pattern (Fig. 4B). Histopathological examination of the resected specimen showed a well-differentiated adenocarcinoma (Fig. 4C).

\section{Case 4: H. pylori-positive gastric cancer}

This patient had a typical irregular microvascular pattern and irregular microsurface pattern (Fig. 5). An irregularly shaped, depressed lesion measuring $14 \mathrm{~mm}$ in diameter was detected on the anterior wall side of the lesser curvature of the middle gastric body under conventional white-light endoscopy (Fig. 5A). A demarcation line recognizable in terms of differences between the microvascular architecture and the microsurface structure was identified using MENBI. Concerning the microsurface structure within the
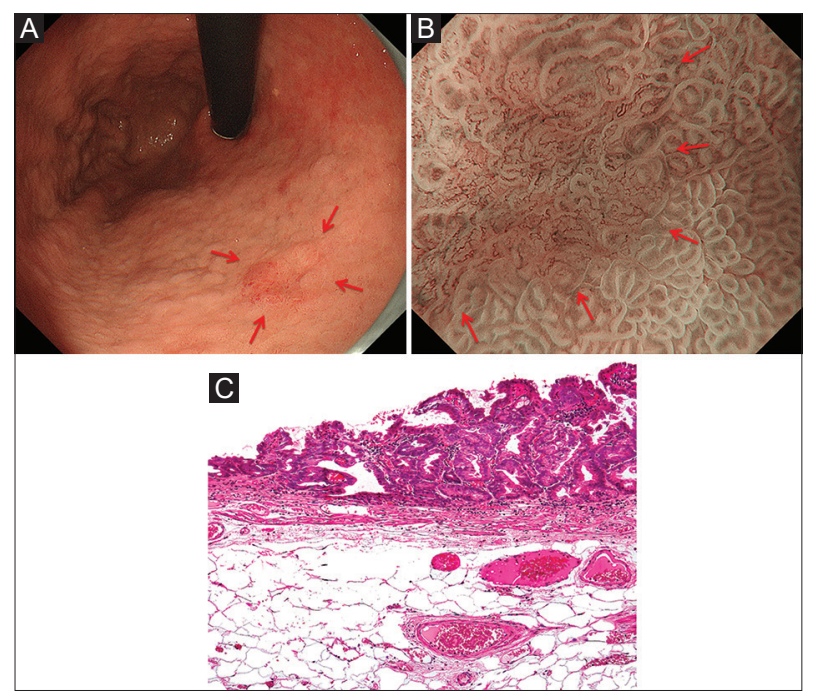

Figure 5 Case 4. Helicobacter pylori-positive gastric cancer: A case of type 0 -IIc early gastric cancer in which the lesion measures $14 \mathrm{~mm}$ and has a typical irregular microvascular pattern and irregular microsurface pattern. (A) Conventional white-light endoscopy: A slightly depressed lesion (red arrow) is found in the anterior wall of the middle gastric body. The margin is unclear. (B) Magnifying endoscopy with narrowband imaging findings. An irregular microvascular pattern and irregular microsurface pattern with a demarcation line are identified. The red arrow indicates the demarcation line. (C) Histopathological findings. The tumor consists of a well-differentiated adenocarcinoma (hematoxylin-eosin stain, $\times 100$ ) 
demarcation line, marginal crypt epithelia were curved to oval and nonuniform in shape with asymmetric distribution and irregular arrangement. Thus, the irregular microsurface pattern was identified. As for the microvascular architecture, the microvessels showed nonuniform shapes, irregular arrangement, and asymmetric distribution, leading to the identification of the irregular microvascular pattern (Fig. 5B). Histopathological examination of the resected specimen showed a well-differentiated adenocarcinoma (Fig. 5C).

\section{Discussion}

Evidence showing the preventive effect of $H$. pylori eradication on gastric cancer has been reported by numerous researchers [9-12]. However, clinical issues related to gastric cancer detected after $H$. pylori eradication have also been pointed out [13,14]. Kamada et al [13] previously reported that most gastric cancers detected after $H$. pylori eradication are characteristically located in mainly middle-third and lower-third region, are $20 \mathrm{~mm}$ or less in diameter, and are macroscopically of depressed type and histologically of the differentiated type. Herein, in the $H$. pylori-eradicated group, lesions measuring $20 \mathrm{~mm}$ or less accounted for $78 \%(21 / 27)$, those present in mainly middle-third and lower-third region accounted for $85 \%$ $(23 / 27)$, and the predominant macroscopic type was the depressed type; thus, our results are consistent with those of previous studies.

Ito et al have reported that $H$. pylori eradication caused unclear borders or flattening of the tumor in the short term, and the superficial layer was covered by non-neoplastic epithelium in $73 \%$ of such lesions [15]. Kobayashi et al compared images obtained by ME-NBI in the successful eradication group and non-eradicated control group, and reported that a "gastritis-like" appearance was frequently observed under ME-NBI in the successful eradication group [3]. We have also encountered some patients with gastric cancer detected after $H$. pylori eradication in whom a correct diagnosis of cancer was difficult to make based on conventional endoscopy and dye-spraying endoscopy using indigo carmine.

In the present study, results of the analysis using the VS classification system showed that the frequencies of the irregular microvascular pattern were $100 \%$ in both the $H$. pylori-eradicated group and $H$. pylori-positive group. On the other hand, the frequency of the irregular microsurface pattern was significantly lower in the $H$. pylori-eradicated group than in the H. pylori-positive group (78\% vs. 95\%). In addition, the sensitivity of the irregular microsurface pattern used as a marker for detecting gastric cancer after H. pylori eradication was significantly lower than that of the irregular microvascular pattern. These results suggest that the microsurface pattern in gastric cancer detected after $H$. pylori eradication is similar in structure to the non-neoplastic epithelium. This similarity may be partly explained by the fact that the superficial layer of the tumor is covered by the non-neoplastic epithelium, as reported by Ito et al [15]. However, it was possible to recognize the subepithelial irregular microvascular pattern of gastric cancer by close observation of the lesion at the maximal magnification of ME-NBI using the water immersion technique. Namely, a correct diagnosis of gastric cancer was successfully made by using the microvascular pattern as a marker. Thus, observation of the lesion at the maximal magnification which can dissect capillaries is required for sufficient diagnostic performance.

The reported causative factors for difficulty in detecting gastric cancer after $H$. pylori eradication include further differentiation in the superficial layer of the tumor [15] and the presence of the epithelium with low-grade atypia [16], in addition to coverage of the non-neoplastic epithelium over the superficial layer of the tumor. Although these factors are reported to be responsible for unclear borders of the lesion, it remains controversial whether these epithelia are non-neoplastic or represent surface differentiation of the cancer or tumor cells with low-grade atypia. High frequencies of cancer with low-grade atypia in cases of gastric cancer detected after $H$. pylori eradication have been reported. Nakagawa et al [17] reported that $H$. pylori eradication induced a series of endoscopic and histological changes including appearance of surface regenerative and lower-grade atypical epithelium. Based on the results of this study, the number and frequency of gastric cancer lesions containing the component of a very well-differentiated adenocarcinoma at 37 (median) months after H. pylori eradication were as follows: 15 (56\%) lesions in the H. pylorieradicated group and $17(29 \%)$ in the $H$. pylori-positive group. Thus, gastric cancer containing the component of a very well-differentiated adenocarcinoma was statistically significantly higher in the $H$. pylori-eradicated group than in the $H$. pylori-positive group $(\mathrm{P}=0.02)$. However, the irregular microvascular pattern was suggested to be useful for diagnosing cancer by magnifying endoscopy, regardless of the presence or absence of the component of a very welldifferentiated adenocarcinoma.

The limitation of this study was that it was a retrospective single-center study. A multicenter prospective study under the same conditions of endoscopic observation will be necessary in the future.

In conclusion, it may be difficult to make a correct diagnosis of early gastric cancer after $H$. pylori eradication by endoscopic procedures. However, the results of this study suggest that identification of the irregular microvascular pattern, rather than the microsurface pattern, based on the VS classification system by ME-NBI at the maximal magnification, is useful for making a correct diagnosis of such gastric cancer. 


\section{Summary Box}

\section{What is already known:}

- It is difficult to detect the gastric cancer after Helicobacter pylori ( $H$. pylori) eradication using endoscopic diagnostic procedures including magnifying endoscopy with narrow-band imaging (ME-NBI)

- Previously report employed the microsurface pattern alone as the main diagnostic marker for making a diagnosis of early gastric cancer after H. pylori eradication, though the diagnostic performance is not optimal

\section{What the new finding is:}

- Another marker of the irregular microvascular pattern which can be visualized by ME-NBI at the maximal magnification, based on the vessels plus surface classification system is remarkably useful for making a correct diagnosis of the gastric cancer after H. pylori eradication

\section{References}

1. Yamaoka Y. How to eliminate gastric cancer-related death worldwide? Nat Rev Clin Oncol 2018;15:407-408.

2. Saka A, Yagi K, Nimura S. Endoscopic and histological features of gastric cancers after successful Helicobacter pylori eradication therapy. Gastric Cancer 2016;19:524-530.

3. Kobayashi M, Hashimoto S, Nishikura K, et al. Magnifying narrowband imaging of surface maturation in early differentiatedtype gastric cancers after Helicobacter pylori eradication. J Gastroenterol 2013;48:1332-1342.
4. Yao K, Anagnostopoulos GK, Ragunath K. Magnifying endoscopy for diagnosing and delineating early gastric cancer. Endoscopy 2009;41:462-467.

5. Muto M, Yao K, Kaise M, Kato M, Uedo N, Yagi K, Tajiri H. Magnifying endoscopy simple diagnostic algorithm for early gastric cancer (MESDA-G). Dig Endosc 2016;28:379-393.

6. Yao K. The endoscopic diagnosis of early gastric cancer. Ann Gastroenterol 2013;26:11-22.

7. Yao K, Uedo N, Kamada T, et al. Guidelines for endoscopic diagnosis of early gastric cancer. Dig Endosc 2020;32:663-698.

8. Yao K, Oishi T, Matsui T, Yao T, Iwashita A. Novel magnified endoscopic findings of microvascular architecture in intramucosal gastric cancer. Gastrointest Endosc 2002;56:279-284.

9. Fukase K, Kato M, Kikuchi S, et al; Japan Gast Study Group. Effect of eradication of Helicobacter pylori on incidence of metachronous gastric carcinoma after endoscopic resection of early gastric cancer: an open-label, randomized controlled trial. Lancet 2008;372:392-397.

10. Maehata Y, Nakamura S, Fujisawa K, et al. Long-term effect of eradication on the development of metachronous gastric cancer after endoscopic resection of early gastric cancer. Gastrointest Endosc 2012;75:39-46.

11. Mabe K, Takahashi M, Oizumi H, et al. Does eradication therapy for peptic ulcer prevent gastric cancer? World J Gastroenterol 2009; 15:4290-4297.

12. Take S, Mizuno M, Ishiki K, et al. The effect of eradicating on the development of gastric cancer in patients with peptic ulcer disease. Am J Gastroenterol 2005;100:1037- 1042.

13. Kamada T, Hata J, Sugiu K, et al. Clinical features of gastric cancer discovered after successful eradication of Helicobacter pylori: results from a 9-year prospective follow-up study in Japan. Aliment Pharmacol Ther 2005;21:1121-1126.

14. Take S, Mizuno M, Ishiki K, et al. The long-term risk of gastric cancer after the successful eradication of Helicobacter pylori. $J$ Gastroenterol 2011;46:318-324.

15. Ito M, Tanaka S, Tanaka S, et al. Morphological changes in human gastric tumours after eradication therapy of in a short-term follow up. Aliment Pharmacol Ther 2005;21:559-566.

16. Kitamura Y, Ito M, Matsuo T, et al. Characteristic epithelium with low-grade atypia appears on the surface of gastric cancer after successful eradication therapy. Helicobacter 2014;19:289-295.

17. Nakagawa M, Sakai Y, Kiriyama Y, et al. Eradication of Helicobacter pylori induces immediate regressive changes in early gastric adenocarcinomas. Pathobiology 2019;86:135-144. 